Med Buccale Chir Buccale 2014:20:73-74

(C) SFCO, 2014

DOI: $10.1051 / \mathrm{mbcb} / 2014012$

\title{
Éditorial
}

\section{MERCI JACKY !}

Après 18 années passées à la SFC0, notre Maître le Professeur Samson, notre Ami Jacky a décidé de mettre fin à ses activités au sein de notre Société. De mes souvenirs de jeune diplômé et assistant, je garde l'image d'un modèle, une sorte de but à atteindre. L'école genevoise qu'il incarnait a tiré vers le haut notre Société, grâce à la présentation d'études prospectives en implantologie, de cas cliniques originaux de médecine et de chirurgie orales ou de conférences magistrales. J'ai le souvenir mémorable du congrès de Toulouse où Jacky avait exposé avec brio les lésions blanches de la muqueuse buccale. Outre la richesse de l'iconographie de cette conférence, il avait déployé toute sa verve pour bannir les notions de leucoplasie et de dysplasie pour les remplacer par kératose et néoplasie orale intra-épithéliale. Outre le débat d'experts que cela peut susciter, cette approche iconoclaste a permis à certains d'entre nous de remettre en question nombre d'idées reçues [1]. Le doute pour arriver à la certitude, cette philosophie que Descartes nous a enseignée, n'est malheureusement pas légion dans le monde médico-chirurgical où l'élève répète ce qu'il a appris du maître qui le tenait d'un maître plus ancien Une longue chaîne qui fait perdurer des notions ou des conduites à tenir inadaptées. Je citerais en pensant à Jacky la chirurgie orale chez les patients sous anti-coagulants. Pendant de nombreuses années nous avons prescrit des relais hépariniques à nos patients pour prévenir un prétendu risque hémorragique alors que l'école genevoise préconisait une hémostase locale efficace, simple à mettre en œuvre. La confrontation de nos avis et les données « Evidence-based » ont remis en cause la pratique du relais chez les plus « intrépides » d'entre nous. Forte de ces expériences, la SFMBCB a édité des recommandations publiées en 2006 [2]. Aujourd'hui, l'attitude préconisée 30 ans plus tôt par l'école genevoise et son leader J. Samson est devenue la règle. Comme un serpent de mer, les vieux démons resurgissent. L'arrivée des nouveaux anti-coagulants plus justement appelés anti-coagulants directs semble remettre en cause ce que nous avons mis plusieurs décennies à comprendre. En chirurgie orale, le risque hémorragique est faible. Lorsqu'il est mis en balance du risque thrombotique, on doit inexorablement conclure qu'une hémostase locale www.mbcb-journal.org

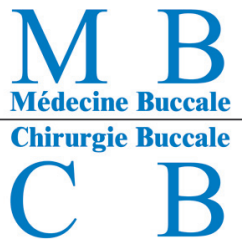

appropriée est le plus souvent suffisante. Toute modification de traitement qui majore le risque thrombotique est une perte de chance pour le patient. Heureusement, les prochaines recommandations de la SFCO, auxquelles a participé le $\operatorname{Pr}$ J. Samson, permettront de ne pas revenir à des pratiques ancestrales.

Comment retracer l'itinéraire de J. Samson dans notre société sans parler de la revue MBCB. Jacky a porté à bout de plume la revue pendant 10 ans. Il a publié 16 éditoriaux, 43 articles et corrigé l'ensemble des publications de la revue. Il a pendant toute cette période assumé le rôle de rédacteur en chef. Sa persévérance, son abnégation au service de la revue ont permis d'avoir dans un premier temps des publications régulières pour arriver à un rythme de croisière de 4 numéros par an depuis 2005. Dans un deuxième temps, il a été décidé de conformer la revue aux exigences uniformes pour les manuscrits soumis aux revues biomédicales [3], avec en ligne de mire une indexation plus visible. Je crois que Jacky aurait aimé partir avec l'indexation Medline «en poche ». Pour le travail accompli pendant toutes ces années, pour l'exemple qu'il a représenté pour notre société, notre revue, nous nous devons de poursuivre le travail accompli. C'est dans cet esprit que je souhaite exercer le rôle de rédacteur en chef de la revue MBCB. Pour m'aider dans cette tâche, j'ai demandé au Pr P. Lesclous de me seconder, ce qu'il a accepté sans grande hésitation, lui aussi mu par «l'esprit » et l'enseignement de J. Samson. En revanche, la pérennité et la qualité de la revue ne dépendent pas exclusivement des rédacteurs en chef, mais aussi des auteurs et des relecteurs qui doivent continuer de s'investir avec encore plus de rigueur et de volonté. La revue est la vitrine de notre Société, de la chirurgie orale. Nous devons la pérenniser dans la continuité de ce qu'a initié le Pr J. Samson. Merci Jacky d'avoir ouvert la voie, d'avoir fait vivre notre revue qui continuera d'exister dans l'esprit que tu as initié : un brin iconoclaste, un rien intransigeant, les pages grandes ouvertes à nos confrères francophones qui participent activement à la vie de $M B C B$.

Jean-Christophe Fricain Rédacteur en chef $M B C B$

\footnotetext{
*Correspondance : fricainj@aol.com
} 


\section{Conflits d'intérêt : aucun}

\section{Références}

1. Küffer R, Lombardi T. Premalignant lesions of the oral mucosa. A discussion about the place of oral intraepithelial neoplasia (OIN). Oral Oncol 2002;38:125-30.
2. Recommandations pour la prise en charge des patients sous anti vitamine $\mathrm{K}$ en chirurgie bucco dentaire. MBCB 2006; 188-212.

3. Exigences uniformes pour les manuscrits soumis aux revues biomédicales: rédaction et édition de la publication biomédicale 2010. http://www.icmje.org/french.pdf 